\title{
ESTIGARRIBIA Bruno et Justin PINTA (dir.), Guarani linguistics in the 21st century
}

Élodie Blestel

\section{OpenEdition}

\section{Journals}

Édition électronique

URL : https://journals.openedition.org/jsa/16025

DOI : 10.4000/jsa. 16025

ISSN : 1957-7842

\section{Éditeur}

Société des américanistes

\section{Édition imprimée}

Date de publication : 15 juin 2018

Pagination : 289-296

ISSN : 0037-9174

\section{Référence électronique}

Élodie Blestel, « ESTIGARRIBIA Bruno et Justin PINTA (dir.), Guarani linguistics in the 27st century », Journal de la Société des américanistes [En ligne], 104-1 | 2018, mis en ligne le 15 juin 2018, consulté le 04 septembre 2022. URL : http://journals.openedition.org/jsa/16025 ; DOI : https://doi.org/10.4000/jsa. 16025

Ce document a été généré automatiquement le 4 septembre 2022

Tous droits réservés 


\title{
ESTIGARRIBIA Bruno et Justin PINTA (dir.), Guarani linguistics in the 21st century
}

\author{
Élodie Blestel
}

\section{RÉFÉRENCE}

ESTIGARRIBIA Bruno et Justin PINTA (dir.), Guarani linguistics in the 21st century, Brill (Brill's studies in the indigenous languages of the Americas, 14), Leiden/Boston, 2017, 421 p., réf. dissém., index, ill. en noir et blanc, tabl., graph.

Guarani linguistics in the 21st century réunit pour la première fois les contributions de chercheurs spécialistes de différents dialectes du guarani (guarani paraguayen pour l'essentiel des travaux mais aussi guarani mbyá et de Corrientes), offrant ainsi un précieux panorama des dernières recherches sur un ensemble dialectal d'environ 8 millions de locuteurs répartis entre le Paraguay, la Bolivie, l'Argentine et le Brésil. Si le statut de chacun de ces dialectes diffère sensiblement selon la situation linguistique des zones concernées - le guarani jouit du statut de langue officielle nationale depuis 1992 au Paraguay, où il est parlé par environ $80 \%$ de la population alors qu'on ne lui accorde qu'une reconnaissance régionale en Bolivie et en Argentine -, il s'agit là d'un cas unique en Amérique puisque la plupart des locuteurs sont d'origine métisse, en particulier au Paraguay, pays qui était encore largement monolingue jusqu'à la moitié du XIX siècle après que le guarani a servi de langue de colonisation et d'évangélisation (Cerno, p. 353). Or la vitalité de ces dialectes reste menacée par la prépondérance symbolique des langues romanes avec lesquelles ils doivent cohabiter : leur très récente officialisation a sans doute constitué une étape capitale au regard de leur histoire, mais on peine encore à réduire sous la bannière d'un "guarani officiel » un paysage linguistique à la fois complexe et contrasté. Or il s'agit ici de l'un des enjeux de cet ouvrage : les nécessaires débats idéologiques, conflits d'intérêts et pressions politiques que génèrent les processus 
de normalisation linguistique font trop souvent l'impasse sur les études fondées sur des corpus de pratiques langagières spontanées. En outre, la chimère d'un guaraniete (guarani pur) élaborée par une poignée de militants et d'Académiciens - lesquels continuent de s'en disputer l'autorité- a pour corollaire malheureux d'aiguiser le sentiment d'extranéité ressenti par les usagers qui affirment ne parler que jopara, ce qui, dans la pratique, relève d'un mélange encore assez peu étudié entre espagnol et guarani selon des logiques référentielles, directives, expressives, phatiques, métalinguistiques et poétiques sans que l'on puisse le circonscrire de façon systématique (Stewart, p. 406-408). Au-delà de la nécessité des études sur les usages, représentations et attitudes vis-à-vis du guarani, il est donc fondamental d'établir - puis de rendre visibles et accessibles - des recherches proprement linguistiques sur ces dialectes, et c'est précisément ce manque que l'ouvrage édité par Bruno Estigarribia et Justin Pinta vient combler. Car il n'y a pas que dans les villes et dans les sphères de prestige que les langues romanes, et en particulier l'espagnol, n'ont cessé de gagner du terrain: les descriptions métalinguistiques mêmes du guarani, souvent imprégnées de la longue et inertielle tradition gréco-latine, pèchent par leurs carences et imperfections, ce qui a des conséquences redoutables sur l'enseignement de la langue et la mise en œuvre d'un quelconque plan de planification linguistique. Aussi, le fait de réunir des études linguistiques - et partant non normatives - sur le guarani dans un seul et même volume est particulièrement bienvenu dans le contexte sociolinguistique et politique mouvementé de ces trente dernières années. Non seulement il fournit une occasion de faire le point sur l'état des recherches actuelles et sur ce qu'il reste à découvrir sur la (les) langue(s) guarani, mais il permet de rendre visible une communauté de chercheurs, qui y trouve du même coup un lieu d'échanges et de dialogues féconds.

2 L'ouvrage s'ouvre sur un chapitre introductif « Introduction » (p. 1-6) dans lequel Bruno Estigarribia présente les raisons qui ont poussé les éditeurs à réunir ces douze contributions; s'il évoque le nombre insuffisant d'études sur le sujet, c'est surtout semble-t-il celles en langue anglaise qui font défaut selon lui : les sources qu'il cite passent en effet sous silence un grand nombre de travaux, certes épars mais bien de nature linguistique; nous renvoyons à ce qui figure dans les bibliographies réunies par Boidin (2009) et Penner (2014) ${ }^{1}$. Cependant, nous rejoignons l'auteur sur le constat de l'existence de nombreux points aveugles dans des pans entiers des études linguistiques: très peu de travaux explorent des données de syntaxe et de pragmatique et on pourrait ajouter plus généralement qu'on ne sait rien de la variation qui existe entre les parlers à l'intérieur d'un dialecte donné et ce, dans tous les champs de la discipline. Le second chapitre, «A grammar sketch of Paraguayan Guarani » (p. 7-85), est très instructif à cet égard puisque Bruno Estigarribia s'efforce d'y livrer une vue d'ensemble des connaissances actuelles sur le guarani paraguayen, ce qui lui permet de mettre le doigt sur de nombreux manques et aberrations dans les études grammaticales existantes, tout en offrant d'intéressantes pistes de recherche. Il invite ainsi à explorer de manière plus systématique le statut des morphèmes du guarani, lesquels, s'ils sont liés, doivent être classés comme affixes ou clitiques et ce, en vue d'y adosser une pratique orthographique plus cohérente. Par ailleurs, il montre combien il est encore difficile pour les chercheurs de se départir des classes de mots romanes pour qualifier ces morphèmes : la distinction nom/verbe fait régulièrement débat - l'affirmation de l'auteur selon laquelle ce problème est en partie résolu pourrait en effet être discutée ${ }^{2}-$, mais il faut encore explorer le problème de la catégorie d'« adverbe » - tout aussi ambigüe pour les langues romanes-, ainsi que celui du statut des morphèmes de temps, aspect, modalité (TAM) 
dont certains ne sont identifiés que comme marqueurs de temps alors qu'on aurait tout intérêt à en étudier la portée déictique et évidentielle (ce à quoi s'emploie plus loin Maura Velázquez-Castillo, voir infra). Enfin, l'auteur constate avec raison combien tout reste à faire en matière de syntaxe théorique : les propriétés syntaxiques qui président aux ellipses, coordinations, extractions, etc., restent encore aujourd'hui totalement méconnues pour ce qui concerne la langue guarani, de même que leurs interfaces avec la sémantique.

3 C'est dans un même souci de rationaliser les descriptions du guarani paraguayen que Shaw Gynan présente, dans un troisième chapitre intitulé «Morphological glossing conventions for the representation of Paraguayan Guarani » (p. 86-130), un inventaire de 70 morphèmes liés pour lesquels il propose d'adapter les principes de Lehmann (2004). L'intention de l'auteur est d'abord de fournir aux chercheurs un système de gloses interlinéaires qui permette de mener des études de corpus à grande échelle - études dont on a souligné combien elles étaient nécessaires, ne serait-ce que comme préalable à la standardisation de la langue - mais cela lui permet ce faisant de soulever le problème des grilles d'analyse que l'on doit utiliser, notamment pour la question des morphèmes liés au verbe dont l'absence de description prosodique empêche la juste caractérisation.

Pour clore ces quatre premiers chapitres à caractère général, Jorge Gómez Rendón s'intéresse enfin à l'émergence du dialecte identifié comme " guarani paraguayen » avec une contribution intitulée "The demographics of colonization in Paraguay and the emergence of Paraguayan Guarani »(p.131-157). L'auteur y expose les circonstances sociales, démographiques et historiques qu'il croit être à l'origine de l'émergence de ce parler. Circonstances qui pourraient être résumées en trois volets: l'importance et la composition de la population guarani au moment de la conquête, l'ampleur et le modèle d'immigration et d'installation précoce des Espagnols au Paraguay et enfin, les modèles de colonisation et d'organisation de la population guarani dans les villes espagnoles et indiennes. Selon l'auteur, ce sont en particulier les pratiques socioculturelles du kuñadazgo et du yanaconato - qui impliquaient la présence de femmes indiennes provenant de différentes chefferies dans les espaces productifs et reproductifs des colonies espagnoles - qui sont à l'origine du bilinguisme sociétal et du mélange linguistique caractéristique de cette koinè guarani apparue très tôt en marge des villes. On déplore avec l'auteur le manque de sources pouvant étayer son propos et il semble par ailleurs que les chiffres avancés en termes de peuplement doivent être comparés à ceux des populations des missions dont on sait qu'elles sont probablement venues se mêler aux populations des campagnes après l'expulsion des Jésuites. Dans quelle mesure la variante de guarani pratiquée dans les Réductions a-t-elle apporté son écot dans ce qui est le guarani paraguayen aujourd'hui ? La question mériterait d'être posée eu égard à l'ampleur du peuplement des missions relativement à celui du reste du territoire à l'époque coloniale.

5 Les quatre chapitres suivants (V à VIII) présentent une série d'études ciblées sur des aspects particuliers de la grammaire guarani.

6 Au chapitre V «Word class switching in Guarani syntax» (p. 158-193), Wolf Dietrich se penche sur la question des classes de mots par un examen de données chronologiques et diachroniques provenant de diverses sources dialectales (guarani de Corrientes, Avá, Kaiowá, guarani bolivien tapieté et mbyá). L'auteur avance que la distinction verbe/nom - qu'il fonde sur le paradigme de marques personnelles qu'on associe aux différents lexèmes - est la distinction la plus importante dans tous les dialectes, même s'il convient 
de la relativiser en raison de la propension de certaines racines à passer d'une classe à l'autre, en particulier dans le cas du marquage hiérarchique des personnes en position d'objet. Pour l'auteur, en effet, ces constructions nominales $(3,2>1$ et $3>2)$ doivent être comprises comme des clauses existentielles pour lesquelles on doit distinguer l'agent sémantique de sa marque - qui correspond dans ces cas de figure à un complément sur le plan syntaxique -, ce qui revient à postuler l'existence de deux types de constructions attributives pour ces compléments, appelées ici attribution "interne » et " externe ». Par ailleurs, l'auteur fait valoir l'existence d'une très petite classe d'adjectifs - dits "syntaxiques »- dans la langue, alors qu'il écarte l'existence d'adverbes. L'analyse s'avère convaincante à ceci près qu'il faut s'accorder sur ce que l'on entend par "verbe " et « nom » étant donné le caractère agglutinant de la langue guarani : on ne peut en effet statuer a priori sur le caractère verbal ou nominal d'un lexème donné, puisque c'est seulement son agencement syntaxique qui permet de le définir.

Dans le chapitre VI "The distribution of implicit arguments in Paraguayan Guaraní » (p. 194-230), Judith Tonhauser étudie de manière très détaillée la distribution des arguments implicites en guarani paraguayen, à savoir les arguments du prédicat qui ne présentent pas de marquage explicite sur le prédicat de la proposition principale (que ce soit par un morphème spécifique, un pronom indépendant, ou un syntagme nominal). À l'issue d'un raisonnement très systématique, l'auteur parvient à montrer qu'en guarani paraguayen, en dehors des cas de non-marquage dus au système hiérarchique du marquage de l'objet $(3,2>1$ et $3>2)$, un argument peut être implicite s'il ne renvoie pas à un proto-patient ou à un proto-destinataire humain et s'il ne s'agit pas d'une information importante du point de vue de la structure de l'information. Elle en conclut que le guarani paraguayen présente des caractéristiques typologiques proches des langues dites « orientées vers le discours » comme le japonais, le russe ou le coréen, même si, dans ces dernières, les arguments implicites ne sont pas soumis à la restriction de personne.

Dans le chapitre intitulé "Comparison and the expression of degree in Mbyá Guaraní » (p. 231-258), Guillaume Thomas propose une analyse des constructions comparatives du guarani mbyá, en vue d'en livrer une compréhension plus approfondie depuis une optique onomasiologique. L'auteur présente des données nouvelles, issues d'un travail d'élicitation auprès de trois locuteurs de Kuña Piru (Misiones). On peut toutefois émettre des réserves quant à la description même des faits linguistiques : on se demande si les verbes ne sont qualifiés comme tels, non pas selon leurs propriétés d'agencement syntaxique, mais selon des critères extra-linguistiques, voire en fonction de leur traduction dans leur versant roman. Il en est de même de la qualification de tel ou tel autre morphème comme "adjectif » ou " adverbe ». Ainsi, l'auteur s'étonne-t-il de la similitude entre l'utilisation du mbyá heta qu'il glose par « beaucoup » (many) et mbovy'i " peu» (few) et de leurs équivalents anglais en ce qu'ils peuvent à la fois entrer dans des structures comparatives et fonctionner comme "adverbes" de quantité. Mais que l'association de heta - que l'on pourrait tout aussi bien gloser par un substantif, par exemple, « quantité »- au morphème inverseur de polarité -ve " plus » puisse établir une comparaison sans importer la quantité effective n'est pas réductible au morphème heta seul : il ne s'agit pas seulement d'une question de morphologie et/ou de sémantique, mais bien, encore une fois, d'une question de syntaxe: seule la succession des deux morphèmes dans la chaîne parlée - et ce, dans un ordre bien précis puisque le standard de comparaison semble toujours précéder ce qui lui est comparé, ce qui n'est 
malheureusement pas clairement formulé dans ce travail - est apte à établir une structure comparative.

Pour clôturer cette section, Maura Velázquez-Castillo examine les marqueurs évidentiels de type rapporté -je/ndaje, aipo, ra'e, raka'e en guarani paraguayen dans un article intitulé «Deixis and perspective in Paraguayan Guaraní reportive evidentiality (p. 259-284), qu'elle présente dans une analyse unifiée et à contre-courant du traitement qui en est fait par la tradition descriptive (on trouve habituellement ces morphèmes parmi les marqueurs déictiques, temporels ou évidentiels). L'auteur parvient de manière très convaincante à faire la distinction entre ce qui relève du signifié paradigmatique imputable à chacun des éléments pris séparément et ce qui émerge de leur accumulation en syntaxe, puisque celle-ci peut donner lieu à des effets discursifs autonomes que l'auteur prend soin d'identifier et de documenter.

Les quatre derniers chapitres du volume portent enfin sur des questions ayant trait au contact guarani-espagnol.

11 Au chapitre IX « Spanish Loans and evidence for stratification in the Guarani lexicon » (p. 285-314), Justin Pinta et Jennifer L. Smith livrent une analyse phonologique des différentes stratégies d'adaptation des emprunts espagnols dans la langue guarani. Selon eux, le lexique de cette dernière est stratifié de manière synchrone dans une structure de type noyau-périphérie selon le degré d'adaptation de chacun des éléments. Cette étude est très novatrice en ce sens qu'elle montre que l'adaptation phonologique des emprunts répond à des logiques non-fortuites et stratifiées. On pourra toutefois objecter que le système phonologique standard sur lequel se fondent les comparaisons ne prend pas en compte les réalisations propres au castillan paraguayen: comparer par exemple l'espagnol fút.bol au guarani hú.vol « football » (p. 293) a ceci de problématique qu'on ne tient pas compte de la possible réalisation labiodentale et fricative fú.vol répandue chez les locuteurs. Autrement dit, étant données l'ampleur du bilinguisme et l'étendue des réalisations phonétiques possibles qui ne se voient pas reflétées dans la graphie, postuler deux ensembles linguistiques discrets (espagnol vs guarani) au sein desquels on observerait le devenir des emprunts donne peut-être une vision seulement partielle des processus en jeu.

Le chapitre $\mathrm{X}$ " Insertion and backflagging as mixing strategies underlying GuaraníSpanish mixed words » (p. 315-347) est consacré aux « mots mixtes ». Bruno Estigarribia y montre que le mélange opère lorsque les bases espagnoles se voient augmentées de morphèmes du guarani, alors que l'inverse ne se produit pas. Par ailleurs, à l'aune de la typologie établie par Muysken (2000, 2013), il avance que différents processus entraînent le mélange de mots guarani : les racines espagnoles dans les clauses guarani apparaissent via un processus d'insertion et les marqueurs phrastiques et quelques autres particules apparaissent dans des clauses non-guarani par un processus connu sous le nom de backflagging (voir Muysken 2013). Enfin, les postpositions de guarani dans les clauses espagnoles se font par un processus de double insertion. Les données présentées invite l'auteur à réitérer l'idée selo laquelle le jopara serait mieux décrit comme un parler mixte (mixed lect), au sens de Backus (2003).

Dans « Aspects of dialectal diversification of Guarani in Paraguay and Corrientes: contact between two given languages in different settings» (p. 348-378), Leonardo Cerno compare les dialectes de guarani parlés au Paraguay et à Corrientes, deux dialectes ayant connu un contact étroit et prolongé avec l'espagnol depuis le milieu du $\mathrm{xVI}^{e}$ siècle. L'auteur entend établir une corrélation entre l'intensité du contact et les changements 
observés sur quelques points précis des deux langues et ce, en adoptant des hypothèses explicatives multilatérales tout en faisant cas des possibles convergences entre guarani et espagnol. Les données de l'auteur sont très intéressantes puisqu'elles tentent de mettre de côté la causalité interne pour prendre en compte à la fois le contact entre les systèmes linguistiques, mais aussi la possible influence de l'histoire des communautés, ainsi que leurs situations sociolinguistiques respectives.

Andrew Stewart clôt le volume avec une contribution intitulée « Jopara and the SpanishGuarani language continuum in Paraguay: considerations in linguistics, education and literature » (p.379-416), dans laquelle il examine la question du jopara dans une perspective à la fois historique, structurelle, politique et socio-linguistique. Bien qu'il s'agisse pour lui davantage d'une pratique langagière que d'un système linguistique, l'auteur montre de façon très pertinente comment la représentation d'un bilinguisme coordonné contraste avec la situation linguistique du pays dans laquelle les pratiques jopara dominent. Il condamne ainsi cet «essentialisme stratégique " qui consiste à éclipser ces pratiques du (des) guarani(s) promu(s) par le ministère de l'Éduction paraguayen (MEC), mais aussi par l'Ateneo de lengua y cultura guarani, l'Académie et même certains écrivains représentant l'élite culturelle, ce qui donne lieu à une polarisation puriste du continuum espagnol-guarani. L'auteur milite à l'inverse pour que les puristes et les locuteurs du jopara trouvent un terrain d'entente entre le guarani rural de certaines propositions littéraires et le parler spontané des locuteurs, en n'omettant pas de se demander quel doit être le rôle futur du guarani dans la société.

Les éditeurs de cet ouvrage de très bonne tenue ${ }^{3}$ ont fait le pari de réunir pour la première fois des spécialistes reconnus pour leurs contributions à la recherche dans le domaine de la linguistique guarani. Le dialogue et les différents renvois entre les chapitres attestent que cette mise en commun était nécessaire; le résultat est réussi.

\section{BIBLIOGRAPHIE}

\section{BACKUS Ad}

2003 " Units in codeswitching: evidence for multimorphemic elements in the lexicon », Linguistics, 41 (1), p. 83-132.

\section{BoIDIN Capucine}

2009 "Esquisse de bibliographie "en" et "sur" le Tupi Guarani », Nuevo Mundo Mundos Nuevos [en ligne], Bibliographies, https://journals.openedition.org/nuevomundo/51373, consultée le $10 / 07 / 2018$.

KALLFELL Guido

2016 « ¿Cómo hablan los paraguayos con dos lenguas? Gramática del jopara », Biblioteca Digital Curt Nimuendajú [en ligne], http://www.etnolinguistica.org/biblio:kallfell-2016-jopara, consultée le $10 / 07 / 2018$.

LEHMANN Christian

2004 «Interlinear morphemic glossing ", in Geert Booij, Christian Lehmann, Joachim Mugdan 
et Stavros Skopeteas (dir.), Morphologie. Halbband 2. Ein internationales handbuch zur flexion und wortbildung, Walter de Gruyter, Berlin/New York, p. 1834-1857.

LIUZZI Silvio

2006 Guaraní elemental. Vocabulario y gramática, Moglia Ediciones, Corrientes.

\section{MUYSKEN Pieter}

2000 Bilingual speech. A typology of code-mixing, Cambridge University Press, Cambridge.

2013 " Language contact outcomes as the result of bilingual optimization strategies ", Bilingualism: Language and Cognition, 16 (4), p. 709-730.

PENNER Hedy

2014 Guaraní aquí. Jopara allá. Reflexiones sobre la (socio)lingüística paraguaya, Peter Lang (Fondo hispánico de lingüística y filología, 19), Bern.

\section{NOTES}

1. Ajoutons, pour rectifier les propos de l'auteur, que la thèse de Guido Kallfell, d'abord rédigée en allemand, est également disponible en langue espagnole : voir Kallfell 2016.

2. Voir notamment la position de Silvio Liuzzi pour lequel cette distinction n'est justement pas pertinente : "Se puede postular que toda unidad léxica, autónoma o dependiente [...], tiene vocación predicativa, por el hecho que en guaraní la oposición verbo-nominal es irrelevante, por un lado, y por otro, el predicado es universal, mientras que el verbo para expresarlo específicamente, es una particularidad de muchas lenguas " (Liuzzi 2006, p. 71).

3. Il manque visiblement quelques exemples p. 219 et il reste quelques coquilles et erreurs de transcription dans les citations du texte de Stewart. Par ailleurs, les résumés des contributions ainsi que l'affiliation et les coordonnées des auteurs auraient été appréciés, mais ce ne sont là que quelques remarques de détail (ou peut-être des suggestions pour un prochain volume?).

\section{AUTEURS}

\section{ÉLODIE BLESTEL}

Université Sorbonne Nouvelle, CLESTHIA (EA 7345) 